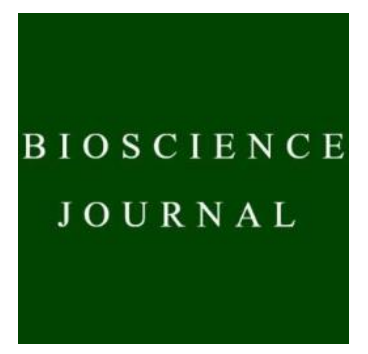

\title{
CLASSIFICATION OF THE SPERM CHROMATIN COMPACTION ALTERATIONS IN BULLS (BOS taUrUS) AND ITS CORRELATION WITH THE EFFICIENCY OF THE IN VITRO PRODUCTION OF EMBRYOS
}

Sara Hissae HIRAIWA ${ }^{1}$ (i), Paulo Henrique Mazzutti ALVES² (i), Bruno Augusto Nassif TRAVENÇOLO ${ }^{3}$ (D), Muller Carrara MARTINS² iD, Marcelo Emílio BELETTI ${ }^{4}$ iD

\footnotetext{
${ }^{1}$ Graduate Program in Cell and Structural Biology, Federal University of Uberlândia, Uberlândia, Minas Gerais, Brazil.

2 Graduate Program in Veterinary Science, Federal University of Uberlândia, Uberlândia, Minas Gerais, Brazil.

${ }^{3}$ Faculdade de Computação, Federal University of Uberlândia, Uberlândia, Minas Gerais, Brazil.

${ }^{4}$ Laboratory of Biology of Reproduction, Federal University of Uberlândia, Uberlândia, Minas Gerais, Brazil.
}

Corresponding author:

Marcelo Emílio Beletti

Email: mebeletti@ufu.br

How to cite: HIRAIWA, S.H., et al. Classification of the sperm chromatin compaction alterations in bulls (Bos taurus) and its correlation with the efficiency of the in vitro production of embryos. Bioscience Journal. 2021, 37, e37028. https://doi.org/10.14393/BJ-v37n0a2021-47967

\begin{abstract}
This paper proposes to classify the sperm chromatin compaction alterations in bulls, according to the affected area location and its objective is evaluating the correlation of the intensity, the heterogeneity and these kinds of chromatin decompaction with the rates of cleavage and the formation of blastocysts of in vitro production of embryos (IVPE). It was used several subfertile animals sperm samples, which were evaluated using the toluidine blue staining and computer image analysis, making possible the categorization of the chromatin decompaction according to their location. The percentages of chromatin decompaction and heterogeneity were also evaluated. IVPEs were done and the rates of cleavage and of blastocysts were correlated with the chromatin characteristics. It made possible the classification of the chromatin decompaction according to the head affected part in at least four types: base decompaction, basal half decompaction, central axis decompaction, total decompaction. Based on the correlation, it can be implicated that each type of classification has different influences on the bull fertility. It made possible understanding that sperms amount with $5 \%$ or more of chromatin decompaction intensity interferes in the bull fertility and this condition can be featured as an uncompensable defect, while the heterogeneity of chromatin is not an important factor in the IVPE results.
\end{abstract}

Keywords: Chromarin. Image analysis. IVF. Sperm analysis.

\section{Introduction}

Sperm are cells with a differentiated structure and specialized in carrying the paternal genetic and epigenetic charge to the oocyte. The condensation of the sperm chromatin is an important phase in the distinction that comes during the spermatogenesis, at about 90 to $95 \%$ of the somatic histones are changed by protamines (Oliva 2006), configuring a highly compacted structure, the toroid of the protamine, which is important for the protection and maintenance of the stability of the DNA (Beletti 2013).

It is believed that the remaining regions of histones can have significant functions in the process of fertilization and embryonic development (Oliva 2006). Gineitis et al. (2000) believe that the histones can be connected to the telomeres, specific structures of the DNA and proteins found at the extremity of the chromosomes (Shammas 2011), and to the first events of the sperms that respond to signs of the oocyte for the formation of the male pronucleus. Furthermore, the histones are important sites of epigenetic signalization and they can be related to the paternal epigenetic heritage (Carrel 2012). 
The failures in the formation of the sperm can induce alterations of the stability of the nuclear chromatin and alter the genetic and epigenetic regulations of the embryonic development (Aitken and de Iuliis 2007; Beletti 2013).

Among the methods of analysis of the sperm chromatin, the analysis Sperm Chromatin Structure Assay $\left(\mathrm{SCSA}^{\circledR}\right)$ that uses the fluorescence of the sperms stained with acridine orange $(\mathrm{AO})$ evaluated by flow cytometry is the more used. Since 1980, hundreds of publications demonstrated the efficiency of the SCSA in the evaluation of male fertility (Evenson 2016).

Another method was created by Mello (1982) for the analysis of the sperm chromatin through the induced metachromasy in a smear of semen stained with Toluidine Blue (TB), a substance capable to combine with the DNA phosphates presenting this phenomenon. To increase the sensitiveness of the technique, a pretreatment with acid is done to partially remove the protamines of the regions with alterations of the chromatin, facilitating the ligation of the TB, increasing the sensitivity of the method. This procedure also allows the morphological and morphometric analysis of each sperm concomitant with the chromatin analysis, and although it is slower, it has a lower cost in relation to SCSA ${ }^{\circledR}$. To reduce the subjectivity and increase accuracy, Beletti et al. (2004 e 2005) developed an algorithm for computational evaluation of the chromatin decompaction using photomicrographs of the smear of the semen stained with TB. This means it was possible to measure the intensity and the homogeneity of the decompaction through all the head. Applying a similar algorithm, Beletti et al. (2004) and Souza et al. (2018) also demonstrated the existence of alterations in the chromatin in different locations and variations in the intensity of the decompaction.

It has already been demonstrated that semen quality, including chromatin alterations, has an influence on the efficiency of in vitro embryo production (Kipper et al. 2017; Siqueira et al. 2018). However, there are some recent articles that question the effect of chromatin alterations on the results of PIVEs (Siqueira et al. 2018; Anbari et al. 2020).

The objective of this study was to evaluate the intensity, the heterogeneity and the location of the chromatin decompaction in bull sperms and correlates these results with the rate of cleavage and the formation of blastocysts in IVPE routines.

\section{Material and Methods}

\section{Materials}

The chemical products, unless those indicated in another way, were acquired from Sigma-Aldrich Chemical Inc. (St. Louis, MO, USA). The means used to the IVPE were produced in the Laboratório de Biologia da Reprodução of Universidade Federal de Uberlândia (Laboratory of Reproduction Biology of the Federal University of Uberlandia).

\section{Collection of samples}

This study was approved by the Comitê de Ética em Pesquisas da Universidade Federal de Uberlândia (Committee on Ethics in Researches of the Federal University of Uberlandia), Brazil, under the number 031/12.

Bulls between 30 and 36 months old, with a good seminal quality (motility $>80 \%$ and morphological pathologies $<30 \%$ ), maintained in semiconfinement in the Fazenda do Glória of Universidade Federal de Uberlândia (Gloria's Farm of the Federal University of Uberlandia), were submitted to scrotal insulation according to Lucio et al. (2016).

A first sample of the semen was collected using an artificial vagina before the insulation process and the others were collected weekly after the scrotal insulation of two days during the 60 days. The samples were patterned to a concentration of 40 million mobile sperms in TRIS-egg yolk diluent and glycerol $6.5 \%$ (Chacur et al. 2012), and loaded into $0.5-\mathrm{mL}$ straws. Cooling was started by transferring straws into a programmable freezing machine that performed (TK 3000, Uberaba, Brazil) the cooling $\left(-0.25^{\circ} \mathrm{C} / \mathrm{min}\right.$ to 5 ${ }^{\circ} \mathrm{C}$ ) and freezing rates $\left(1^{\text {st }}\right.$ step: $-15^{\circ} \mathrm{C} / \mathrm{min}$ to $-80{ }^{\circ} \mathrm{C} ; 2^{\text {nd }}$ step: $-10^{\circ} \mathrm{C} / \mathrm{min}$ to $\left.-120{ }^{\circ} \mathrm{C}\right)$. Subsequently, the straws were plunged into liquid nitrogen. It is important to emphasize that the semen samples before 
insulation presented a good and they presented various modifications, including the chromatin compaction, after the insulation, as shown by Lucio et al. (2016).

Semen samples of a normalizing bull, of a central of semen, previously tested and with good results in the IVPE were also used.

For the IVPE routines, cow ovaries were collected in a cold-storage room, maintained in a saline solution with an antibiotic (Gentamicin $50 \mu \mathrm{g} / \mu \mathrm{L}$ ) under a temperature of 36 to $38^{\circ} \mathrm{C}$ and transported to the laboratory. The oocytes of follicles with a diameter between 3 and $8 \mathrm{~mm}$ were aspirated and kept in a tube of $50 \mathrm{~mL}$ in a water bath under a temperature of $38.5^{\circ} \mathrm{C}$, transferred to a disposable petri dish of $100 \times 20 \mathrm{~mm}$ and evaluated. Nearly 6,200 oocytes with at least four layers of cells from cumulus and homogeneous cytoplasm, rated as grades I and II (Lucio et al. 2016) were selected and washed in a solution with TCM 199 with Hank's salts $(8.55 \mathrm{mg} / \mathrm{mL})$, Gentamicin $(50 \mu \mathrm{g} / \mu \mathrm{L}), 10 \%$ Fetal Calf Serum (FCS), HEPES acid $(2.16 \mathrm{mg} / \mathrm{mL})$, HEPES sodium $(2.34 \mathrm{mg} / \mathrm{mL})$ and sodium bicarbonate $(0.38 \mathrm{mg} / \mathrm{mL})$. The half part of these oocytes was used for fertilization with bull semen with insulated testicles and the other half with the semen of the normalizing bull.

The evaluation of the cleavage (number of cleaved embryos/number of oocytes that underwent the maturation) was done during day 2 (D2) after the fertilization. The evaluation of the embryo development until the blastocysts stage (number of blastocysts/number of cleaved embryos) was analyzed 7 days after the fertilization (D7). Before the application of the statistical tests, as a way to partially neutralize the effects of the not controllable variables in the IVPE, it was done the normalization of the data acquired with the semen of insulated animals, having as reference the results acquired by the normalizing animal, previously tested in our laboratories and proven fertile. For this purpose, the rates of cleavage and of blastocysts of the samples of insulated bulls were transformed in the percentage of the rates of cleavage and blastocysts acquired with the normalizing bull semen in the same routine of IVPE, according to the equations:

$$
\begin{gathered}
\text { normalised rate of cleavage }=\frac{\text { cleavage rate of insulated bull }}{\text { cleavage rate of normalizing bull }} \\
\text { normalised rate of blastocysts }=\frac{\text { blastocyst rate of insulated bull }}{\text { blastocyst rate of normalizing bull }}
\end{gathered}
$$

\section{In vitro production}

The oocytes were separated into groups of 20 matured in drops of $100 \mu \mathrm{L}$, made of TCM-199 $(8.55 \mathrm{mg} / \mathrm{mL})$ supplemented with $10 \%$ fetal calf serum $(F C S)$, sodium bicarbonate $(1.98 \mathrm{mg} / \mathrm{mL})$, Gentamicin $(50 \mu \mathrm{g} / \mu \mathrm{L}), 0.5 \mu \mathrm{g} / \mu \mathrm{L} \mathrm{FSH}$ (Folltropin- $\mathrm{V}^{\circledR}$ ) and $5 \mu \mathrm{g} / \mu \mathrm{L} \mathrm{LH}\left(\right.$ Lutropin $^{\circledR}$ ) disposed in a petri dish of $60 \times 30 \mathrm{~mm}$ and covered with mineral oil. The petri dish was preincubated under a temperature of $38.5{ }^{\circ} \mathrm{C}, 5 \% \mathrm{CO} 2$ and $95 \%$ humidity for 22 to 24 hours.

The straws of semen were thawed under a temperature of $38.5^{\circ} \mathrm{C}$ in water bath for $40 \mathrm{~s}$. After thawing, the semen went through selection in Percoll gradient of $90 \%(350 \mu \mathrm{L})$ and $45 \%(350 \mu \mathrm{L})$ in microtubes of $1.5 \mathrm{~mL}$ and centrigation for 7 minutes at 9,000 $\times$ G. The precipitate was transferred to another microtube of $1.5 \mathrm{~mL}$ with $500 \mu \mathrm{L}$ of a TALP-Sperm medium and centrifuged for 3 minutes at 9,000 $\times \mathrm{G}$ to remove the Percoll waste of the sperms (Folchinl et al., 2012). An aliquot of $30 \mu \mathrm{L}$ from the precipitate was resuspended in a medium of fertilization (IVF medium) which consisted of TALP supplemented with PHE ( $2 \mathrm{mM}$ penicillin, $1 \mathrm{mM}$ hypotaurine, $245 \mu \mathrm{M}$ epinephrine) and $0.6 \mathrm{UI} / \mathrm{mL}$ heparin.

On the day of fertilization (D0) the matured oocytes were washed twice in IVF medium, transferred to drops of $90 \mu \mathrm{L}$ of IVF medium in a fertilization plate and subsequently covered with mineral oil. Approximately 20 oocytes were placed in a drop and added a volume of semen adjusted to $1 \times 106$ mobile sperms $/ \mathrm{mL}$ of IVF medium. They were coincubated at $38.5^{\circ} \mathrm{C}, 5 \% \mathrm{CO} 2$ and humidity of $95 \%$ for 18 hours.

One day after the fertilization (D1), to mechanical removal of the cells of the cumulus of the zygotes, there were used successive pipetting in the drops. They were washed twice with a synthetic oviduct fluid (SOF), supplemented with a solution of essential amino acids 50X (BME) and non-essential 100X (MEM), $340 \mu \mathrm{M}$ trisodium citrate, $2.8 \mathrm{mM}$ Myo-inositol, $5 \mathrm{mg} / \mathrm{mL}$ bovine serum albumin (BSA) and $2.5 \%$ of FCS in 4- 
well culture dish covered with mineral oil. Subsequently, they were incubated at $38.5^{\circ} \mathrm{C}, 5 \% \mathrm{CO} 2$ and $95 \%$ humidity. On the third day (D3) after fertilization, a part of the culture medium was changed and the embryos were kept incubated until the seventh day (D7) after fertilization.

\section{Sperm analysis}

During the preparation of the semen for the IVPE, a drop of semen before and after the Percoll selection was used to make the smears. Those were fixed in ethanol acetic acid 3:1 for one minute, followed by $70 \%$ ethanol for three minutes and dried at room temperature. They were hydrolyzed in $4-\mathrm{N}$ hydrochloric acid for 20 minutes and washed in detailed water. After they were air-dried, the smears were stained with 0.025\% Toluidine Blue (TB), pH 4.0 in a phosphate citric acid buffer (Mcllvaine's buffer) on the slide (Beletti et al. 2005). When the coverslip was placed over the slide, it waited 3 minutes and digital images were taken of about 100 sperms per lamina, using the optical microscope Leica DM500 with objective of 100x (immersion), coupled at a Leica ICC50 camera. The sperm heads were segmented through an algorithm, developed in the SCILAB computational environment. Succeeding, the images of the heads were evaluated by another algorithm, in which there were obtained the average pixel value that made up each head. The software delineated the modifications of the chromatin taking into account the heads considered normal. These heads were automatically selected by the program, considering the average of the pixels of the six brightest (more compacted) and homogeneous heads.

The heads of the samples were evaluated according to the decompaction place on the basis of the study of Beletti et al. (2004), being classified into five types: base decompaction (BD), basal half decompaction (BHD), central axis decompaction (CAD), total decompaction (TD) and dispersed areas (DA) (Figure 1).

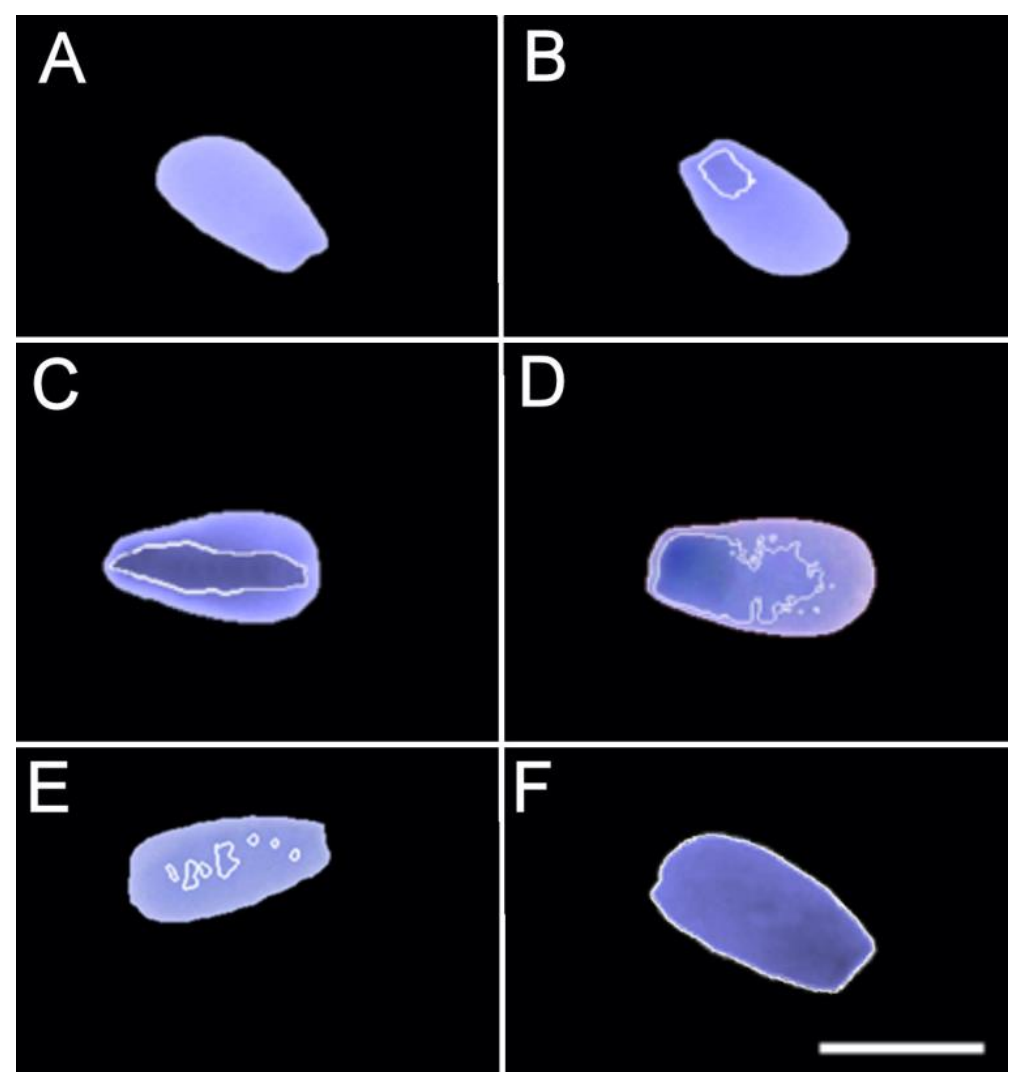

Figure 1. Image output from software that identifies each type of sperm chromatin decompaction. A head of sperm with normal chromatin; $B$ - base decompaction (BD); $C$ - central axis decompaction (CAD); $\mathrm{D}$ - basal half decompaction (BHD); $\mathrm{E}$ - dispersed areas (DA); $\mathrm{F}$ - total decompaction (TD). Barr $=5 \mu \mathrm{m}$.

They were also evaluated about the intensity of the chromatin decompaction (Dec), considered as the difference between the average pixel value of each analyzed head in relation to the average of the standard heads and subsequently transformed into a percentage of the average pixel value for the standard heads. The heads were also evaluated about the heterogeneity of the chromatin (Het), according to the 
coefficient of variation of the gray-level (pixel value) calculated for each head in percentage. To a quantitative evaluation of these modifications there were used three approaches, and the average of decompaction and heterogeneity in all the sperms that were analyzed in the evaluated sample, as the percentage of carrier sperms of decompaction and/or heterogeneity over two levels of alterations, 5 and $10 \%$ of the evaluated sample (Beletti et al. 2005).

IVPE was evaluated according to their percentage of zygotes that cleaved on D2 and the percentage of cleaved embryos that developed until the blastocyst stage on D7 and correlated with the percentage of sperms that suffered from each type of modifications of the chromatin classified as the location of the decompaction in the nucleus of the sperm and the intensities of the decompaction and heterogeneity.

\section{Statistical methods}

It was done the Pearson correlation test between the variables. It was thought to exist correlation when the coefficient had a value of $p<0.05$ and with inclination to have correlation when the value was $0.05<p<0.10$ (Pomini et al. 2015). All the statistical analysis was done using the S-plus program (MathSoft, Inc.; 2000, Cambridge, MA, USA).

\section{Results}

Among the samples of semen collected before and after the scrotal insulation, only 50 presented motility and viability to be employed in the IVPE routines.

There were done 50 IVPE routines using 3,136 grades I and II oocytes for each group of semen. The rate of cleavage when using the semen of an insulated bull was $49.71 \%(1,559 / 3,136)$ and the rate of a confirmed fertile bull was $69.32 \%(2,174 / 3,136)$. The rate of blastocysts was $14.88 \%(232 / 1,559)$ for IVPE using the semen of insulated bull and $47.01 \%(1,022 / 2,174)$ for the confirmed fertile bull.

After the selection of the sperm by the Percoll technique, it was not possible to analyze some smears, because these presented technique artifacts that appeared as small dots on and around the heads (Figure 2), which had made it impossible for the automatic segmentation of the heads and when the segmentation was possible, many times these dots were identified as chromatin alteration.

Figure 2. Smear of sperm after Percoll selection staining by toluidine blue showing drops on sperm heads, which can be mistaken for decompacted areas. Barr $=5 \mu \mathrm{m}$.

\section{Location of the chromatin alterations}

The percentage of the chromatin alterations were measured in the sperms before and after the Percoll selection according to their location (Table 1). After the selection the alterations increased and appeared a type that was not classified before the Percoll selection, dispersed areas (DA), that possibly occurred due to the technique artifacts induced by Percoll. 
The results of IVPE were correlated with the percentage of each type of chromatin alteration (Table 2). Only the CAD and the TD had a significant and negative correlation with the rate of cleavage in the IVPE, while the normal sperms had a significant and positive correlation with the rate of cleavage for those evaluated before the Percoll selection. The classes BD and BMD presented a tendency to negative correlation with the rate of cleavage.

After the Percoll selection, the values of the correlations for the most part were close to zero, including the results correlated with the normal sperms. Only the TD presented a tendency to negative correlation with the rate of blastocysts.

Table 1. Percentage of each chromatin alterations before and after Percoll selection.

\begin{tabular}{ccc}
\hline Chromatin alteration & Before Percoll & After Percoll \\
\hline BD & $10.46 \pm 11.95$ & $12.38 \pm 11.48$ \\
BHD & $9.75 \pm 8.25$ & $23.69 \pm 17.38$ \\
CAD & $2.62 \pm 2.84$ & $4.03 \pm 4.84$ \\
TD & $0.77 \pm 0.85$ & $3.71 \pm 3.53$ \\
DA & - & $6.78 \pm 4.05$ \\
Normal & $76.40 \pm 19.77$ & $49.41 \pm 25.47$ \\
\hline
\end{tabular}

Data are the means \pm s.d. of all semen samples. Base decompaction (BD), basal half decompaction (BHD), central axis decompaction (CAD), total decompaction (TD) and dispersed decompacted areas (DA).

Table 2. Correlation coefficients between each chromatin alteration and the rates of cleavage and blastocysts in IVPE.

\begin{tabular}{|c|c|c|c|c|}
\hline \multirow{2}{*}{ Chromatin alteration } & \multicolumn{2}{|c|}{ Before Percoll } & \multicolumn{2}{|c|}{ After Percoll } \\
\hline & Cleavage & Blastocysts & Cleavage & Blastocysts \\
\hline $\mathrm{BD}$ & $-0.27 * *$ & $-0.25^{* *}$ & -0.08 & -0.05 \\
\hline BHD & $-0.27^{* *}$ & -0.23 & 0.03 & -0.22 \\
\hline CAD & $-0.31 *$ & 0.07 & 0.06 & -0.06 \\
\hline TD & $-0.29 *$ & -0.20 & 0.04 & $-0.28 * *$ \\
\hline DA & - & - & -0.03 & -0.06 \\
\hline Normal & $0.35^{*}$ & 0.24 & 0.00 & 0.23 \\
\hline
\end{tabular}

${ }^{*} p<0.05 ;{ }^{* *} 0.05<p<0.10$. Base decompaction (BD); basal half decompaction (BHD); central axis decompaction (CAD); total decompaction (TD); dispersed areas (DA).

\section{Chromatin heterogeneity and decompaction}

The evaluations of chromatin heterogeneity and decompaction are demonstrated in table 3.

The indexes of the chromatin heterogeneity and decompaction were correlated with the results of the IVPE (Table 4). Before the selection using the Percoll technique, it was verified a significant negative correlation of the cleavage with three levels of decompaction (Dec). These same levels presented a negative correlation with the formation of blastocysts, but not significant. After the Percoll selection, all the parameters evaluated by the Dec were close to zero. For the heterogeneity evaluation, it was not observed a significant correlation with the rates of cleavage as well as the formation of blastocysts.

Table 3. Heterogeneity and decompaction of the sperm chromatin before and after Percoll selection.

\begin{tabular}{ccc}
\hline Chromatin Characteristic & Before Percoll & After Percoll \\
\hline Average Het & $7.72 \pm 4.90$ & $8.24 \pm 4.14$ \\
Het $>5 \%$ & $51.28 \pm 23.75$ & $62.27 \pm 19.74$ \\
Het $>10 \%$ & $25.29 \pm 25.06$ & $31.35 \pm 24.67$ \\
Average Dec & $3.47 \pm 1.61$ & $6.58 \pm 1.69$ \\
Dec $>5 \%$ & $16.44 \pm 22.27$ & $72.46 \pm 22.51$ \\
Dec $>10 \%$ & $2.47 \pm 6.97$ & $12.40 \pm 18.08$ \\
\hline
\end{tabular}

Data are the means \pm s.d. of all semen samples. Average Het - the average percentage of heterogeneity of all the spermatozoa; Het $>5 \%$ - the percentage of spermatozoa with heterogeneity superior to $5 \%$; Het $>10 \%$ - the percentage of spermatozoa with 
heterogeneity superior to $10 \%$; Average Dec - the average percentage of decompaction of all the spermatozoa; Dec $>5 \%-$ the percentage of sperms with decompaction superior to $5 \%$; Dec $>10 \%$ - the percentage of spermatozoa with decompaction superior to $10 \%$.

Table 4. Correlation coefficients between sperm chromatin heterogeneity and decompaction and the rates of cleavage and of blastocysts in IVPE.

\begin{tabular}{ccccc}
\hline Chromatin & \multicolumn{2}{c}{ Before Percoll } & \multicolumn{2}{c}{ After Percoll } \\
\cline { 2 - 5 } alteration & Cleavage & Blastocysts & Cleavage & Blastocysts \\
\hline Average Het & -0.03 & 0.09 & 0.03 & -0.16 \\
Het $>5 \%$ & -0.14 & 0.00 & -0.06 & -0.16 \\
Het $>10 \%$ & 0.00 & 0.09 & -0.07 & $-0.27^{* *}$ \\
Average Dec & $-0.40^{*}$ & -0.17 & 0.12 & 0.08 \\
Dec $>5 \%$ & $-0.34^{*}$ & -0.16 & 0.01 & 0.01 \\
Dec $>10 \%$ & $-0.36^{*}$ & -0.11 & 0.02 & -0.01 \\
\hline
\end{tabular}

${ }^{*} p<0.05 ; * * 0.05<p<0.10$. Average Het - the average percentage of heterogeneity of all the spermatozoa; Het $>5 \%-$ the percentage of spermatozoa with heterogeneity superior to $5 \%$; Het $>10 \%$ - the percentage of spermatozoa with heterogeneity superior to $10 \%$; Average Dec - the average percentage of decompaction of all the spermatozoa; Dec $>5 \%$ - the percentage of sperms with decompaction superior to $5 \%$; Dec $>10 \%$ - the percentage of spermatozoa with decompaction superior to $10 \%$.

\section{Discussion}

There are several methodologies used to evaluate the chromatin and the SCSA ${ }^{\circledR}$ has been the more used (Evenson 2016) because it enables the evaluation of a large number of sperms in a quick manner. The technique of staining with TB is a simpler and cheaper method, which enables to evaluate the morphology and morphometry concomitantly with the sperm chromatin. The association with the computational analysis minimizes the subjectivity and enables morphometric and chromatin evaluations of each sperm. Therefore, it was possible the classification of the alterations of chromatin compaction according to the location at the head of the sperm (Souza et al. 2018).

Considering only the results before the Percoll selection, the types of modification of the chromatin more frequently found were BD and BMD. The basal region of the head of the sperm is where it is situated the nuclear annulus (Ward and Coffey 1989), a region where the sequences of the DNA are not condensed by toroids of protamine and and can be more susceptible to decompaction (Beletti et al. 2004). The alterations of CAD and TD, which can be considered more serious because they reach a great area of the head of the sperm, appeared with less frequency.

When correlating the changes with the IVPE results, it can be inferred, due to the positive correlation of the sperm bearer of normal chromatin with the rate of cleavage, that the fertilization among various factors, also depends on full chromatin. This can be explained because the changes in the chromatin compaction are related to the morphological alterations (Nagy et al. 2013) since the heads of the sperms are basically made of chromatin (Beletti 2013). The alterations of CAD and TD had a negative correlation, and $\mathrm{BD}$ and $\mathrm{BMD}$ tended to a negative correlation with the rate of cleavage, confirming that their presence interferes considerably in the process of fertilization of the oocyte. In relation to the formation of blastocysts, there were no significant correlations with the modifications examined. These data must consider that the rate of blastocysts was calculated from the structure that started the cleavage and not in relation to the number of oocytes originally used. Therefore, the blastocysts formed probably did not appear from oocytes that had been fertilized by sperm with these modifications. Or, when the chromatin modifications are not serious enough to alter the fertilizing capacity of the sperm, they also would not interfere in the initial embryonic development.

Correlating the results of the Dec of sperm before the Percoll selection with the results of the IVPE, it can be inferred that the average decompaction of the sperms of the sample is a good indicator of fertility problems because they have a significant negative correlation with the rate of cleavage.

The correlation between the rate of cleavage and the number of sperms with decompaction bigger than 5 and 10\%, enables us to infer that the sperms with these percentages of decompaction can be considered anomalous because they have a negative correlation with the rate of cleavage. Kipper et al. (2017) did not verify a relation between the number of sperms with more than $2 \%$ of decompaction and the 
results of the IVF. But, with the results of this study, it can be inferred that the presence of sperm with chromatin decompaction bigger than $5 \%$ can be considered an uncompensable defect (Saacke. 2008) and it cannot be tolerated in levels bigger than 15-20\% (Barth and Oko 1989). In relation to the formation of blastocysts, there are no significant correlations in any of the three tested conditions: average decompaction of the sperm and amount of sperm with more than 5 or $10 \%$ of decompaction intensity. Silva et al. (2008) using bull semen with high fertility checked that small variations in the chromatin compaction have a small influence in the cleavage and normal embryo development. In the same way, Kipper et al. (2017) did not observe the influence of the sperm chromatin compaction in the IVF results, but hitherto there were no studies that verify the influence of the more intense chromatin decompaction on the IVF results.

Concerning Het, there have not been significant correlations with the IVPE results. In this case, the staining variation, that represents the different chromatin compaction intensity in the same head, do not correlate with the rate of cleavage and blastocyst formation, that is, it does not influence the initial embryonic fertilization and development, characterized as a little relevance to identify fertility problems.

Changes in the chromatin compaction can be the morphological expression of epigenetic changes, as residual histones in abnormal quantity and/or location and DNA methylation in inadequate levels. Therefore, it is important to emphasize that the effects of the alterations evaluated in this study can be due to epigenetic problems and understanding this mechanism, it is possible to contribute to the understanding of the paternal effects in the embryonic development process (Carrel 2012). With this in mind, it is known that the residual sperm histones are associated with the transcriptional control, chromatin compaction and DNA methylation, and they influence the oocyte fertilization (Yamauchi et al. 2011); that the epigenetic patterns of DNA methylation, that are modifications that occur during the phase of the distinction of the spermatogenesis (Trasler 1998), regulate the histones ligation and the chromatin compaction (Waggoner 2007); that inadequate paternal methylation make the embryo development (Yamauchi et al. 2011) and it can lead to pregnancy loss (El Hajj et al. 2011) and that some abnormalities in the methylation of the specific genes are associated to infertility and morphological defects in the sperms (Kumar et al. 2013), all these phenomena can be expressed as alterations in the chromatin compaction.

The selection of sperm is a widely used procedure in the IVPE, but some studies reveal that this technique can lead to alteration in the DNA integrity. The centrifugation, a phase of the Percoll selection, can increase the formation of oxygen reactive species by leukocytes and not feasible cells, elevating the chance of sperm DNA damage (Sakkas and Alvarez 2010). This could explain the identification of a new type of chromatin alteration after the Percoll selection, classified as DA. However, it is more likely that this type of alteration is a technique artifact motivated by Percoll. When using this selection technique, the analysis of the laminas with semen smears was complicated by the presence of microspheres, probably silica particles of Percoll, around and many times over the sperm heads. Many times, they made the computational analysis not viable and for this reason many smears were discarded. Even the apparently normal smears, in the image analysis could have perceptible artifacts only seen by the data processor. Possibly consecutive washes after the selection could avoid these artifacts, however, this hypothesis came to mind only when it was not possible to do them. Thus, it can be concluded that the data showed after the Percoll selection are not reliable.

The evaluation of the chromatin modification before the Percoll selection is important because it can be an indicative parameter of fertility to be included in routine semen analysis. On the other hand, it is important to emphasize that these evaluations are only suggestive of how the chromatin alterations interfere with the fertilizing capacity of the sperm and in the initial embryonic development since the IVF process was used only samples that passed through the Percoll selection. Therefore, the evaluation after the Percoll selection could be even more significat to determine these inferences, only if it had been performed appropriately and with reliable results.

This experiment was done in controlled conditions and culture media and its results cannot be totally extrapolated to the natural conditions of the field, because in these conditions other biological processes are involved in fertilization and embryonic development. 


\section{Conclusions}

The computational evaluation of the bovine semen smears stained with toluidine blue enables the classification of the chromatin decompaction according to the region of the head affected, and each type of classification has different influences in the bull fertility.

The average of the sperm chromatin decompaction of a sample of semen is a good indicator of bull fertility. A simple evaluation of the heterogeneity of the chromatin decompaction is not an important factor to the IVPE results and for the fertility in bulls.

Sperms with $5 \%$ or more of the chromatin decompaction interfere in bull fertility and this condition can be characterized as an uncompensable defect and it cannot be tolerated in levels bigger than 15-20\%.

Authors' Contributions: HIRAIWA, S.H.: acquisition of data, analysis and interpretation of data, drafting the article; ALVES, P.H.M. and MARTINS, M.C.: acquisition of data; TRAVENÇOLO, B.A.N.: acquisition of data, analysis and interpretation of data; BELETTI, M.E.: conception and design, analysis and interpretation of data, drafting the article. All authors have read and approved the final version of the manuscript.

Conflicts of Interest: The authors declare no conflicts of interest.

Ethics Approval: Approved by Research Ethics Committee of Federal University of Uberlândia. Number: 031/12.

Acknowledgments: The authors would like to thank the funding for the realization of this study provided by the Brazilian agencies CNPq (Conselho Nacional de Desenvolvimento Científico e Tecnológico - Brasil), CAPES (Coordenação de Aperfeiçoamento de Pessoal de Nível Superior - Brasil), Finance Code 001, and FAPEMIG (Fundação de Amparo à Pesquisa do Estado de Minas Gerais), Finance Code APQ-01499-12.

\section{References}

AITKEN, R.J. and DE IULIIS, G.N. Origins and consequences of DNA damage in male germ cells. Reproductive BioMedicine Online. 2007, 14(6), 727-33. https://doi.org/10.1016/S1472-6483(10)60676-1

ANBARI, F., et al. Does sperm DNA fragmentation have negative impact on embryo morphology and morphokinetics in IVF programme? Andrologia. 2020, 52(11), e13798. https://doi.org/10.1111/and.13798

BARTH, A.D. and OKO, R.J. Abnormal Morphology of Bovine Spermatozoa. Ames: lowa State University Press, 1989.

BELETTI, M.E. Cromatina espermática: quebrando paradigmas. Revista Brasileira de Reprodução Animal. 2013, 37(2), 92-6. http://cbra.org.br/pages/publicacoes/rbra/v37n2/pag92-96\%20(RB465).pdf

BELETTI, M.E., COSTA, L.F. and GUADIEIRO, M.M. Morphometric features and chromatin condensation abnormalities evaluated by toluidine blue staining in bull spermatozoa. Brazilian Journal of Morphological Science. 2005, 22(1), 85-90.

BELETTI, M.E., COSTA, L.F., VIANA, M.P. A computational approach to the characterization of bovine sperm chromatin alterations. Biotechnic and histochemistry. 2004, 79(1), 17-23. https://doi.org/10.1080/10520290410001700774

CARREL, D.T. Epigenetics of the male gamete. Fertility and Sterility. 2012, 97(2), 267-274. https://doi.org/10.1016/i.fertnstert.2011.12.036

CHACUR, M.G.M. et al. Efeito de meios diluentes na viabilidade de sêmen congelado bovino. Veterinária e Zootecnia. 2012, 19(1), 346-55.

EL HAJJ, N. et al. Methylation status of imprinted genes and repetitive elements in sperm DNA from infertile males. Sexual Development. 2011, 5(2), 60-69. https://doi.org/10.1159/000323806

EVENSON, D.P. The Sperm Chromatin Structure Assay (SCSA (R)) and other sperm DNA fragmentation tests for evaluation of sperm nuclear DNA integrity as related to fertility. Animal Reproduction Science. 2016, 169, 56-75. https://doi.org/10.1016/j.anireprosci.2016.01.017

FOLCHINI, N.P., et al. Uso de mini-Percoll modificado para seleção e redução da formação de espécies reativas de oxigênio (ROS) em espermatozoides bovinos. Revista Brasileira de Reprodução Animal. 2012, 36(4), 239-44.

GINEITIS, A.A., et al. Human sperm telomere-binding complex involves histone H2B and secures telomere membrane attachment. The Journal of Cell Biology. 2000, 151(7), 1591-98. https://doi.org/10.1083/jcb.151.7.1591

KIPPER, B.H., et al. Sperm morphometry and chromatin condensation in Nelore bulls of different ages and their effects on IVF. Theriogenology. 2017, 87(1), 154-60. https://doi.org/10.1016/j.theriogenology.2016.08.017

KUMAR, M., et al. Novel insights into the genetic and epigenetic paternal contribution to the human embryo. Clinics. 2013, 68(Suppl.1), 5-14. https://doi.org/10.6061/clinics/2013(Sup01)02

LUCIO, A.C., et al. Selected sperm traits are simultaneously altered after scrotal heat stress and play specific roles in in vitro fertilization and embryonic development. Theriogenology. 2016, 86(4), 924-33. https://doi.org/10.1016/j.theriogenology.2016.03.015

MELLO, M.L.S. Induced metachromasia in bull spermatozoa. Histochemistry, 1982, 74(3), 387-92. https://doi.org/10.1007/BF00493438

NAGY, S., et al. Sperm chromatin structure and sperm morphology: their association with fertility in Al-dairy Ayrshire sires. Theriogenology.

2013, 79(8), 1153-61. https://doi.org/10.1016/j.theriogenology.2013.02.011 
OLIVA, R. Protamines and male infertility. Human Reproduction Update. 2006, 12(4), 417-35. https://doi.org/10.1093/humupd/dml009

POMINI, P.R.F., et al. Effects of FGF10 on bovine oocyte meiosis progression, apoptosis, embryo development and relative abundance of developmentally important genes in vitro. Reproduction in Domestic Animals. 2015, 50(1), 84-90. https://doi.org/10.1111/rda.12452

SAACKE, R.G. Sperm morphology: Its relevance to compensable and uncompensable traits in semen. Theriogenology. 2008, 70(3), 473-78. https://doi.org/10.1016/j.theriogenology.2008.04.012

SAKKAS, D. and ALVAREZ, J.G. Sperm DNA fragmentation: mechanisms of origin, impact on reproductive outcome, and analysis. Fertility and Sterility. 2010, 93(4), 1027-36. https://doi.org/10.1016/j.fertnstert.2009.10.046

SHAMMAS, M.A. Telomeres, lifestyle, cancer, and aging. Current Opinion in Clinical Nutrition and Metabolic Care. 2011, 14(1), p.28-34. https://doi.org/10.1097/MCO.0b013e32834121b1

SILVA, R.T., MENDES, J.J.O.B. and BELETTI, M.E. Compactação da cromatina e morfometria da cabeça de espermatozoides na produção de embriões "in vitro" utilizando touros zebuínos. Acta Scientiarum Animal Science. 2008, 30(4), 473-8.

https://doi.org/10.4025/actascianimsci.v30i4.1002

SIQUEIRA, A.F.P., et al. Sperm traits on in vitro production (IVP) of bovine embryos: Too much of anything is good for nothing. Plos One. 2018, 13(7), e0200273. https://doi.org/10.1371/journal.pone.0200273

SOUZA, E.T., et al. Sperm chromatin alterations in fertile and subfertile bulls. Reproductive Biology. 2018, 18(2), 177-81. https://doi.org/10.1016/j.repbio.2018.04.001

TRASLER, J.M. Origin and roles of genomic methylation patterns in male germ cells. Seminars in Cell and Developmental Biology. 1998, 9(4), 467-74. https://doi.org/10.1006/scdb.1998.0225

WAGGONER, D. Mechanisms of disease: epigenesis. Seminars in Pediatric Neurology. 2007, 14(1), 7-14. https://doi.org/10.1016/i.spen.2006.11.004

WARD, W.S. and COFFEY, D.S. Identification of a sperm nuclear annulus: a sperm DNA anchor. Biology of Reproduction. 1989, 41(2), 361-70. https://doi.org/10.1095/biolreprod41.2.361

YAMAUCHI, Y., SHAMAN, J. and WARD, W.S. Non-genetic contributions of the sperm nucleus to embryonic development. Asian Journal of Andrology. 2011, 13(1), 31-5. https://doi.org/10.1038/aja.2010.75

Received: 08 April 2019 | Accepted: 23 December 2020 | Published: 10 June2021

This is an Open Access article distributed under the terms of the Creative Commons Attribution License, which permits unrestricted use, distribution, and reproduction in any medium, provided the original work is properly cited. 